\title{
Fracture of an Osteochondroma of Humerus by Arm Tourniquet Inflated to Measure Blood Pressure in Pediatric Patient With Radial Nerve Neuropraxia
}

\author{
Hakan Sarman ${ }^{\mathrm{a}, \mathrm{d}}$, Cengiz Isik ${ }^{\mathrm{a}}$, Murat Bilgi ${ }^{\mathrm{b}}$, Yasin Emre Kaya ${ }^{\mathrm{a}}$, Abdullah Alper Sahin ${ }^{\mathrm{a}}$, Bunyamin Koc ${ }^{\mathrm{c}}$, \\ Ismail Boyraz ${ }^{\mathrm{c}}$
}

\begin{abstract}
Pedicle fracture of an osteochondroma with radial nerve neuropraxia is a rare complication. The aim of this study was to evaluate a case of fractured osteochondroma after the arm tourniquet inflated to measure blood pressure that caused low energy pedicle fracture with radial nerve neuropraxia in a child. The patient was treated with total excision without complication, and its presentation was similar to that of low energy pedicle fracture with radial nerve neuropraxia.
\end{abstract}

Keywords: Child; Tourniquet; Fracture; Osteochondroma; Neuropraxia

\section{Introduction}

Osteochondroma is the most common benign bone tumor [1]. Osteochondromas are seen more in adolescents or children than infants or newborns $[1,2]$. This tumor is defined as a cartilage-capped bony projection arising on the external surface of bone [1]. Osteochondroma is often asymptomatic, but the only clinical symptom is a painless slow-growing mass on the involved bone [1,3]. When compression, fracture, bone deformity and mechanical joint problems occur with symtoms $[1,3]$. In addition, surgical treatment is considered because of pain, disturbance of growth, decreased range of motion, cosmetic, symptoms secondary to compression of anybody organ, fracture and malign transformation [2-7].

In this case report, we discuss an acute pedicle fracture of

Manuscript accepted for publication December 03, 2014

aDepartment of Orthopedics and Traumatology, Abant Izzet Baysal University School of Medicine, 14280, Golkoy, Bolu, Turkey

${ }^{b}$ Department of Anesthesiology and Reanimation, Abant Izzet Baysal University School of Medicine, 14280, Golkoy, Bolu, Turkey

'Department of Physical Medicine and Rehabilitation, Abant Izzet Baysal University School of Medicine, Bolu, Turkey

${ }^{\mathrm{d}}$ Corresponding Author: Hakan Sarman, Department of Orthopedics and Traumatology, Abant Izzet Baysal University School of Medicine, 14280, Golkoy, Bolu, Turkey. Email: hakansarman@yahoo.com

doi: http://dx.doi.org/10.14740/jmc2015w humerus by an osteochondroma with radial nerve neuropraxia, resulting in a pain and low motion. This fracture occurred in a boy, and its presentation was similar to that of low energy pedicle fracture with radial nerve neuropraxia.

\section{Case Report}

A 13-year-old boy presented to the outpatient orthopedics clinic with pain in his right arm after the arm tourniquet inflated to measure blood pressure by orthopedic nurses. On physical examination, there was localized tenderness over proximally and posterior medially aspect of his arm with palpation. Joint of shoulder and elbow range of motion were normal. Vascular examination revealed no abnormality. There was radial nerve injury after the arm tourniquet inflated while motor function

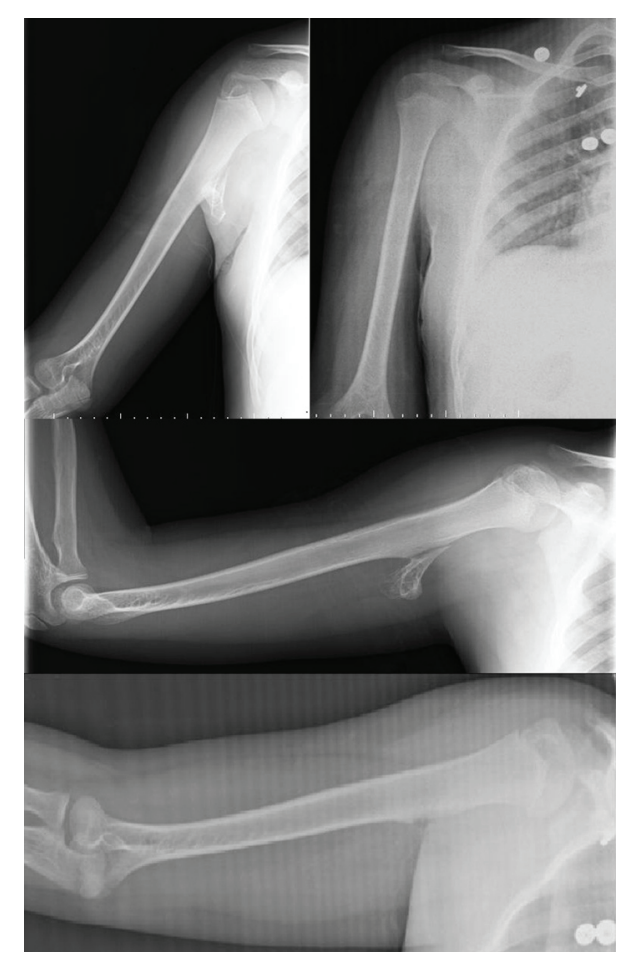

Figure 1. The preoperative and postoperative of radiography of patient. 


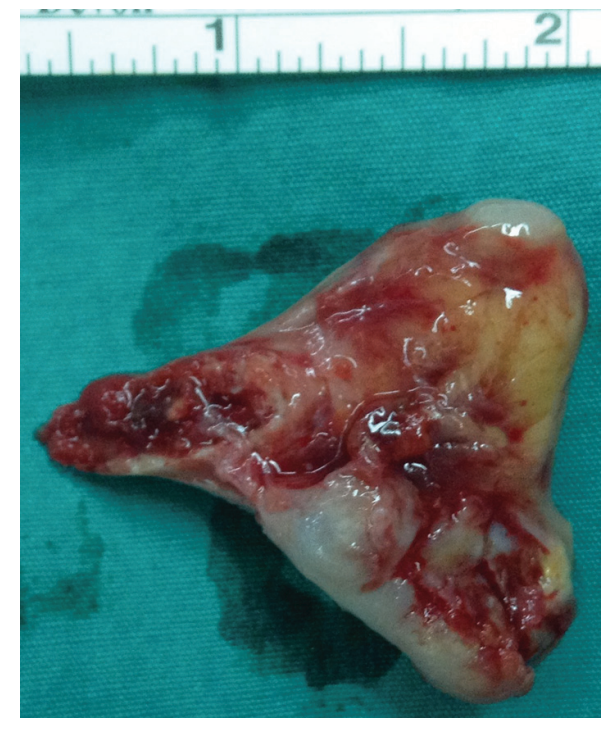

Figure 2. The material of total excision of osteochondroma.

was normal, but there was hypoesthesia on the hand dorsum. His past medical history was not remarkable. Soft tissue injury was initially doubted. Direct radiography showed a pedicle fracture of an osteochondroma on the one-third of the proximal metaphyseal-diaphyseal junction of humerus (Fig. 1). The operation was carried out under general anesthesia. The position of the patient was semi-supine. The approach was done on the right arm through an approximately $5 \mathrm{~cm}$ longitudinal midline posterior-laterally incision. Firstly the exposure was found between biceps and triceps inter-muscular septum, and then achieved the fracture of osteochondroma. It was seen that triceps muscle was injured by pedicle of osteochondroma. Total excision of the osteochondroma was performed without complication (Fig. 1, 2). The diagnosis of osteochondroma was confirmed after pathological examination of the removed part (Fig. 3). The postoperative period was quiet, and the patient has returned to his preoperative level of activity within 2 weeks. The radial nerve deficit was completely healed within 6 weeks.

\section{Discussion}

Osteochondromas are osteocartilaginous exostoses tumors [2, 4]. Osteochondroma occurs in 3\% of the general population. It accounts for $30-49 \%$ of all benign bone tumors and $10-15 \%$ of all bone tumors $[3,8]$. It is typically characterized by metaphyseal location of the long tubular bones, and it is rare on the diaphyseal or the epiphysis $[2,3,5,6]$. Epidemiologically $70 \%$ of affected patients occur at 20 years or younger, and the male to female ratio is $2: 1$ or $1: 1[2,6,8]$. In our case, it was localized in metaphyseal-diaphyseal junction of humerus, and the age of patient was similar in the literature.

Although osteochondromas are generally asymptomatic, it can occasionally come to clinical attention for various reasons $[2,3]$. While our case had swelling, it was not painful before it was fractured. He did not receive any treatments.

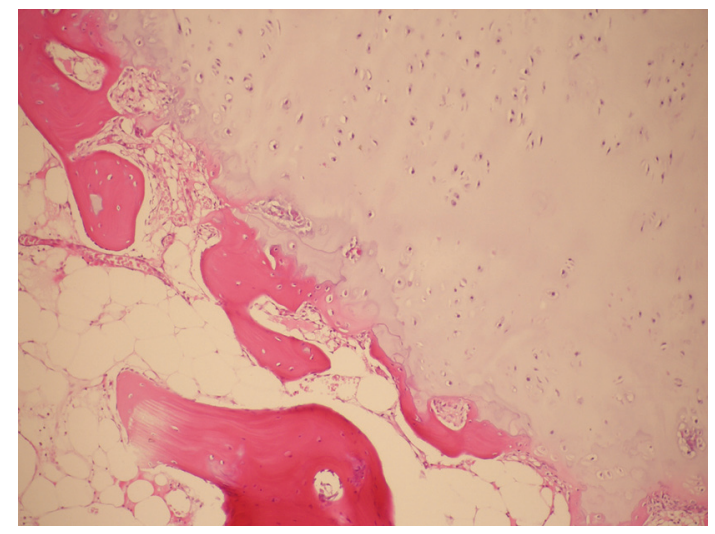

Figure 3. The pathological examination of osteochondroma.

The symptoms are painful swelling, cosmetic problems, restricted motion, nerve or vascular compression and pathological fracture $[2,3,8]$. In the literature, for pathological fracture, bone fracture was more than pedicle fracture $[2,4,6,7]$. The fracture of our case was pedicle.

If symptoms of patients are typical of fracture, it seems local pain and swelling. Initial pain later subsided considerably $[1,8]$. Although the literature contains about 20 issued cases of fracture of an osteochondroma, it is unclear. Because more cases are asymptomatic, these were not published $[1,8]$. The fracture of pedicle of osteochondroma with radial nerve neuropraxia is a rare complication $[1,7,9,10]$. The case was a rare complication after the arm tourniquet inflated to measure blood pressure that caused low energy pedicle fracture with radial nerve neuropraxia in the pediatric ages.

The treatment of fracture of pedicle is divided into conservative or surgical. While conservative authors offer the observation that has a long time pain and more analgesic, surgical authors offer excision that provides faster recovery and return to normal daily activity $[2,3,7]$. We preferred the surgical excision for the case, so he had painful swelling and reduced to extension mechanism. There was no any complication about surgery. After the surgery, the patient was suggested to strengthen for extension mechanism; thus he faster recovered and returned to normal daily activity in the 2 weeks.

Kose et al [7] published low energy fracture of distally metaphyseal of humerus without muscle injured. Reize et al [5] published non-traumatic subscapularis tear, where the tendon had been peeled off the lesser tuberosity by osteochondroma of a young man. Lee et al [6] published restricted motion within a shoulder osteochondroma. Our case was different from others by the localization of diaphyseal [5-7]. Besides, the case was distinguished to have muscle injury [7], faster recovery and returned to normal daily activity from others [5, 6]. Follow-up showed that the radial nerve deficit completely healed within 6 weeks $[9,10]$.

This case report should lead the novice medicine clinician. The case was a rare complication after the arm tourniquet inflated to measure blood that caused low energy pedicle fracture with radial nerve neuropraxia pressure with pediatric ages. The treatment of osteochondroma is very simple. In additional, fracture may be the first symptom of an osteochondroma, and 
we recommend immediate surgical excision without complication.

\section{Conflicts of Interest}

The authors did not receive any grants or outside funding in support of their research or preparation of this manuscript. The authors did not have any financial and personal relationships with other people or organizations that could inappropriately influence (bias) their work.

\section{References}

1. Kitsoulis P, Galani V, Stefanaki K, Paraskevas G, Karatzias G, Agnantis NJ, Bai M. Osteochondromas: review of the clinical, radiological and pathological features. In Vivo. 2008;22(5):633-646.

2. Bottner F, Rodl R, Kordish I, Winklemann W, Gosheger G, Lindner N. Surgical treatment of symptomatic osteochondroma. A three- to eight-year follow-up study. J Bone Joint Surg Br. 2003;85(8):1161-1165.

3. Eyesan SU, Idowu OK, Obalum DC, Nnodu OE, Abdulkareem FB. Surgical consideration for benign bone tumors. Niger J Clin Pract. 2011;14(2):146-150.

4. Koenig SJ, Toth AP, Martinez S, Fletcher JW, Goldner RD. Traumatic pseudoaneurysm of the brachial artery caused by an osteochondroma, mimicking biceps rupture in a weightlifter: a case report. Am J Sports Med. 2004;32(4):1049-1053.

5. Reize F, Buess E. Humeral osteochondroma causing a subscapularis tear: a rare source of shoulder dysfunction. Arch Orthop Trauma Surg. 2007;127(1):67-70.

6. Lee JY, Lee S, Joo KB, Lee BG, Baik SS, Bae J. Intraarticular osteochondroma of shoulder: a case report. Clin Imaging. 2013;37(2):379-381.

7. Kose O, Ertas A, Celiktas M, Kisin B. Fracture of an osteochondroma treated successfully with total excision: two case reports. Cases J. 2009;2:8062.

8. Carpintero P, Leon F, Zafra M, Montero M, Berral FJ. Fractures of osteochondroma during physical exercise. Am J Sports Med. 2003;31(6):1003-1006.

9. Yamamoto T, Tanaka K, Nagira K, Marui T, Akisue T, Kurosaka M, Mizuno K. Intermittent radial nerve palsy caused by a humeral osteochondroma: a case report. J Shoulder Elbow Surg. 2002;11(1):92-94.

10. Coenen L, Biltjes I. High radial nerve palsy caused by a humeral exostosis: a case report. J Hand Surg Am. 1992;17(4):668-669. 